\title{
Solid-Phase Fluorescence and Ionization Efficiency in Negative-Ion Matrix-Assisted Laser Desorption/Ionization of Neutral Oligosaccharides: Interaction Between $\beta$-Carboline Matrix and Ammonium Salt
}

\author{
Tohru Yamagaki, Hiroaki Suzuki, and Kazuo Tachibana \\ Department of Chemistry, School of Science, The University of Tokyo, Tokyo, Japan
}

\begin{abstract}
Ammonium chloride $\left(\mathrm{NH}_{4} \mathrm{Cl}\right)$ with a $\beta$-carboline harmine (7-methoxy-1-methyl-9H-pyrido[3,4$b$ ]indole) Matrix promotes the generation of chloride-anionized molecules of neutral oligosaccharides in negative-ion ultraviolet matrix-assisted laser/desorption ionization mass spectrometry (MALDI MS). The relative abundances of anionized molecules and matrix ([Analyte $+\mathrm{Cl}^{-} /$ [matrix $+\mathrm{Cl}^{-}$) correlate with the amount of $\mathrm{NH}_{4} \mathrm{Cl}$ added, and saturate at a level of $\mathrm{NH}_{4} \mathrm{Cl}$ approximately four times that of the matrix. Their solid-phase fluorescence spectra indicate that harmine and $\mathrm{NH}_{4} \mathrm{Cl}$ form a complex of the hydrochloride salt in the mixed crystal. The peak intensity at $448 \mathrm{~nm}$ from the harmine hydrochloride in the mixed crystal rises logarithmically with the amount of $\mathrm{NH}_{4} \mathrm{Cl}$ added, a result that quantitatively correlates with increases in the ion abundance ratios of the chloride-anionized molecules to that of harmine. The solid-phase spectroscopic method is useful for studying changes in the characteristics of the matrices and additives in the mixed crystal. Harmine hydrochloride, rather than harmine, works as an effective matrix. The attachment of the chloride to the matrix is essential for the generation of chlorinatedanionized molecules in MALDI. An $\mathrm{N}$-acetyl glucosamine residue (GlcNAc) in lacto- $\mathrm{N}$-tetraose promotes the generation of their chloride-anionized molecules, however, multi-GlcNAc residues in N-acetylchitooligosaccharides hinder it. (J Am Soc Mass Spectrom 2007, 18, 714-723) @ 2007 American Society for Mass Spectrometry
\end{abstract}

$\mathrm{M}$ atrix-assisted laser desorption/ionization mass spectrometry (MALDI MS), like electrospray and fast-atom bombardment MS, has been widely used in the structural analyses of carbohydrates. Neutral oligosaccharides are typically examined in the positive-ion mode, whereas acidic oligosaccharides are analyzed in the negative-ion mode. In MALDI, almost all of the measurements of neutrals are made in the positiveion mode because neutral oligosaccharides readily complex to alkali metal ions [1,2]. Even contaminating amounts sodium are likely to attach to analytes in MALDI under conventional measurement conditions in ion-exchanged water. In some cases, investigators add small amounts of alkali metal chlorides to promote their ionization, but the addition of large amounts of salts suppresses ionization not only in MALDI, but also in other ionization methods. In contrast, negative-ion mode measurements of neutral oligosaccharides are rarely performed in MALDI. An unusual matrix $\beta$-carbolin is needed for deprotonated molecules to be gen-

Published online January 22, 2007

Address reprint requests to Dr. T. Yamagaki, Department of Chemistry, School of Science, The University of Tokyo, 7-3-1 Hongo, Bunkyo-ku, Tokyo 113-0033, Japan. E-mail: yamagaki@chem.s.u-tokyo.ac.jp erated, and anionic adducts of the whole molecules were not observed $[3,4]$. Both ions are labile. MALDI process imparts more than enough energy to dissociate deprotonated molecules and anionic adduct $[3,4]$.

New matrix systems, matrices, and anionic dopants themselves have been investigated for their possible use in the negative-ion mode MALDI MS of neutral oligosaccharides. Breuker et al., [5] using binary tetrabutylammonium (TBA) salt/silicon matrices, were able to generate halide ion attachment under special conditions in MALDI. Liquid TBA salts supply halide anion and silicon particulates that work as a UV-absorbing matrix. Nonami et al. [6-8] employed $\beta$-carboline (9H-pyrido[3,4- $b$ ]indole) matrices and observed deprotonated carbohydrate molecules. Halide-anionized molecules were also generated through the use of matrices containing a small amount of ammonium halides [3]. Wong et al. [9, 10] used hydrogen sulfate and alkylsulfonate as anion dopants for oligosaccharides in MALDI in conjunction with conventional matrices such as 2,5-dihydroxyacetophenone (DHAP) and 2,4,6-trihydroxyacetophenone (THAP), and detected the sulfate adducts $\left[\mathrm{M}+\mathrm{HSO}_{4}\right]^{-}$.

In MALDI, structural analyses of neutral oligosaccharides can be performed by means of data from 
postsource decay (PSD) spectra and product-ion spectra resulting from collision induced dissociation (CID). The PSD and CID from sodium-cationized molecules take place predominantly at the glycosyl bond, and are useful for the sequential analysis of sugar chains. However, sugar chains have a structural complexity stemming from different linkage types and branching pattern. Methods for distinguishing these structural isomers are needed to identify their structures. Recently, it was reported that systematic structure analyses of neutral lactooligosaccharides, including linkage and branching isomers, can be performed by means of negative-ion electrospray ionization (ESI) ion-trap and quadrupole/time-of-flight (Q-TOF) mass spectrometry $[11,12]$. The fragmentations derived from negatively charged precursor ions depend on whether they are 3-linked and/or 4-linked $N$-acetylglucosamine residues. In contrast, as described above, the negative-ion mode MALDI MS has not been extensively applied to this task.

We previously studied the negative-ion mode MALDI of neutral oligosaccharides using $\beta$-carboline matrices. We found that the deprotonated molecules $\left([\mathrm{M}-\mathrm{H}]^{-}\right)$with a reducing terminal are labile and decompose easily, [13] and that in-source decay at the reducing terminal depends on the molecules' glycosyl bond types. These results are useful for linkage analysis [14]. The chlorideanionized molecules were stable enough to allow the measurement of their postsource decay spectra that brought us the structural information, including 3and/or 4-linked branching and fucose-substitution positions, so that we could obtain detailed structural information for the lactooligosaccharides as well as their ESI MS measurements [15].

It is important to understand the generation of the chloride-anionized neutral molecules in MALDI because we could develop and establish the analytical methods for the negative ion mode of MALDI MS. As a preliminary step, we optimized the deposition methods [16] and studied the amounts of ammonium chloride needed as an additive to promote the formation of chloride-anionized molecules $\left([\mathrm{M}+\mathrm{Cl}]^{-}\right)$. Cole et al. [4, 17-19] used ESI MS to study the affinity of chloride anions for neutral oligosaccharides [17-19], and the generation and decomposition of chloride-anionized molecules in MALDI MS [4]. Only a small, often comparable amount of ammonium chloride to that of the analyte was required for the generation of chlorideanionized molecules in ESI [18], and the existing conditions were thought to be efficient in MALDI [4]. However, the measurement conditions were not optimized; furthermore, the relationship between the ammonium chloride and the matrix in forming chlorideanionized molecules of neutral oligosaccharides in the MALD ionization were not thoroughly studied.

Analyses of oligodeoxynucleotides and phosphopeptides were also performed by negative-ion MALDI MS with co-matrices such as ammonium salts and polyamines with a view to improving their signal detection [20-26]. The term "co-matrices," which is used in these articles and in this study, refer to non-UVabsorbing compounds added to the mixed crystal of matrices at the molar ratio of ca. 1:1. Co-matrices alone would not work as matrices in MALDI; however, they enhance the functions of the matrix. Currie and Yates [20] reported that the negative-ion yields of oligodeoxynucleotides by using conventional matrices such as 2,5-dihydroxybenzoic acid and 3-hydroxy-4methoxybenzaldehyde were enhanced by ammonium citrate at a molar ratio of 1:1. Pieles et al. [21] showed that the addition of diammonium hydrogen citrate to a trihydroxyacetophenone matrix is an effective combination for reducing cation adducts and improving ion production of the oligodeoxynucleotides. Limbach et al. [22-24] examined organic base co-matrices such as triethylamine, imidazole, piperidine, and polyamine. It was thought that these additives reduced the cation adduction problem and improved the gas-phase stability of the oligodeoxynucleotides. Cheng and Chan [25] reported that ammonium halides work as co-matrices for a 2-amino-5-nitropyridine matrix. All of the above researchers observed the matrix systems' deprotonation of the deprotonated molecules of oligodeoxynucleotides $\left([\mathrm{M}-\mathrm{H}]^{-}\right)$by their matrix system.

Since oligodeoxynucleotides and phosphopeptides are multiply negative-charged molecules, the cation adduction by $\mathrm{Na}^{+}, \mathrm{K}^{+}$, etc. to their phosphate groups severely suppressed their MALD ionization. Large amounts of volatile ammonium ions, working as counter cations of the negative-charged analytes in the mixed crystal system, reduced and eliminated the cation adduction problem in the multiply negative-charged analytes [26]. Their deprotonated molecules $[\mathrm{M}-\mathrm{H}]^{-}$were clearly detected [20-26]. The ionization of oligodeoxynucleotides could differ from that of neutral oligosaccharides; however, the addition of ammonium salts improves the generation of their anionized molecules. Cole et al. $[4,19]$ studied the ionization of neutral and acidic oligosaccharides from the viewpoint of their relative basicity between analytes and hydrohalogens; they used this approach because the halogen anions could coordinate to the hydroxyl group of neutral oligosaccharides and the complex $[\mathrm{M}-\mathrm{H} \cdots \mathrm{Cl}]^{-}$was formed.

In this study, using solid-phase fluorescence spectroscopy, we have focused our attention on the relationship between additives and matrices, and on the roles of the additives in the generation of the halogen anionized molecules of neutral oligosaccharides in the negative ion mode MALDI MS.

\section{Experimental}

\section{Mass Spectrometry}

All MALDI-TOF mass spectra were acquired with an AXIMA-CFR instrument (Shimadzu-Kratos Corp., Kyoto, Japan) equipped with a pulsed $\mathrm{N}_{2}$ laser $(337 \mathrm{~nm})$, at an acceleration energy of $20 \mathrm{kV}$ using 
pulsed delayed extraction. After finding out the irradiation threshold power to detect chloride cationized molecules of the neutrals, the laser power was fixed at the same value through a series of experiments. Each spectrum represents an average of 200 laser shots. All MALDI mass spectra without a specifically described matrix are measured with harmine as a matrix.

\section{Sample and Reagents}

Cellohexaose $\left(\mathrm{Glc} \beta 1 \rightarrow[4 \mathrm{Glc} \beta 1 \rightarrow]_{4} 4 \mathrm{Glc} ; 990.9 \mathrm{Da}\right)$, maltotriose to maltoheptaose (Glc $\alpha 1 \rightarrow[4 \mathrm{Glc} \alpha 1 \rightarrow]_{\mathrm{n}} 4 \mathrm{Glc}, n=$ $1 \sim 5,504.4,666.6,828.7,990.9$, and $1153.0 \mathrm{Da})$, gentiohexaose $\left(\mathrm{Glc} \beta 1 \rightarrow[6 \mathrm{Glc} \beta 1 \rightarrow]_{4} 6 \mathrm{Glc} ; 990.9 \mathrm{Da}\right)$, and isomaltohexaose $\left(\mathrm{Glc} \alpha 1 \rightarrow[6 \mathrm{Glc} \alpha 1 \rightarrow]_{4} 6 \mathrm{Glc} ; 990.9 \mathrm{Da}\right), \mathrm{N}$ acetylchitohexaose $\left(\mathrm{GlcNAc} \beta 1 \rightarrow[4 \mathrm{GlcNAc} \beta 1 \rightarrow]_{4} 4 \mathrm{GlcNAc}\right.$; $1237.2 \mathrm{Da})$ were purchased from Seikagaku-Kogyo, Tokyo, Japan. Lacto- $N$-tetraose $(\mathrm{Gal} \beta 1 \rightarrow 3 \mathrm{GlcNAc} \beta 1 \rightarrow$ $3 \mathrm{Gal} \beta 1 \rightarrow 4 \mathrm{Glc}$ ) was purchased from Sigma-Aldrich, St. Louis, MO. Cellohexitol was prepared by the reduction of cellohexaose with sodium borohydride. All analyte structures are shown in Table 1.

Norharmane (9H-pyrido[3,4- $b$ ]indole) and its hydrochloride salt and harmaline (1-methyl-7-methoxy-3,4dihydro-9H-pyrido[3,4- $b$ ]indole) hydrochloride were purchased from Sigma-Aldrich, MO; harmaline and harman (1-methyl-9H-pyrido[3,4-b]indole) from Wako Chemicals, Japan; and harmine (7-methoxy-1-methyl$9 H$-pyrido[3,4-b]indole) and its hydrochloride salt from Tokyo Chemical Industry, Tokyo, Japan. Ammonium chloride $(99.998 \%)$ and ammonium fluoride $(99.99+\%)$ were purchased from Sigma-Aldrich; ammonium bromide (99\%) from Wako Chemicals. Distilled and ionexchanged water by Milli-Q (Millipore Co., Billerica, MA) and methanol for HPLC grade (Wako Chemicals) were used as solvents.

\section{Mixed Crystal Preparation on the Sample Target}

Sugar chain samples were dissolved in Milli-Q water at a final concentration of $1 \mathrm{mM}$. Among all the matrices, the solubility of harmine was lowest in methanol; the concentration of the saturated solution was estimated from the weight of the dried-up matrix in an aliquot solution $(28.3 \mathrm{mM}, 6.0 \mathrm{mg} / \mathrm{mL})$. The concentrations of all matrices were unified at $28.3 \mathrm{mM}$ in methanol so that their ionization efficiency could be compared with that of harmine. Ammonium chloride aqueous solutions were prepared with Milli-Q water at final concentrations of $11.8 \mathrm{mM}$ (ca. $0.2 \mathrm{eq} \mathrm{mol}$ to matrix in $0.5 \mu \mathrm{L}$ ), 23.6 $\mathrm{mM}$ (ca. 0.4 eq mol to matrix), $47.1 \mathrm{mM}$ (ca. 0.8 eq mol to matrix), $94.2 \mathrm{mM}$ (ca. 1.7 eq mol to matrix), $184.4 \mathrm{mM}$ (ca. 3.3 eq mol to matrix), $282.6 \mathrm{mM}$ (ca. 5 eq mol to matrix), $376.8 \mathrm{mM}$ (ca. $6.7 \mathrm{eq}$ mol to matrix), $471.0 \mathrm{mM}$ (ca. 8.3 eq mol to matrix).

Mixed crystals on the target spots were prepared via a sandwich method [7, 16, 27]; a $0.5 \mu \mathrm{L}$ aliquot of the matrix was deposited and dried, after which $0.5 \mu \mathrm{L}$ aliquots of the analyte solution and ammonium chlo- ride additive were similarly processed. Subsequently, another aliquot of matrix $(0.5 \mu \mathrm{L})$ was also deposited on the mixed crystal thin layer and dried.

Previously, we surveyed three depositing methods in an evaluation of the ionization efficiency of chlorideanionized molecules: the droplet method, the thin-layer method, and the sandwich method described above [16]. The sandwich method was the most effective for the generation of such molecules.

\section{Estimation of Ionization Efficiency}

All spectra were processed using instrumental software, and the spectra were smoothed by a Gaussian function at the instrumental software value of 100 and a baseline correction at the instrumental software value of 100. The ionization efficiency was estimated from the ratio (\%) of the abundance of chloride anionized analyte molecules to matrix molecules ([Analyte $+\mathrm{Cl}^{-} /[\mathrm{Ma}-$ trix $+\mathrm{Cl}]^{-}$). All data points represent an average of 10 measurements of the different spots prepared identically and separately; their standard deviations are shown as error values.

\section{Liquid-Phase Fluorescence and Ultraviolet Spectra}

Fluorescence spectra were acquired with a RF-1500 fluorophotometer (Shimadzu, Kyoto, Japan) at a 300 $\mathrm{nm}$ excitation wavelength using a quartz crystal cell $(10 \times 10 \times 40 \mathrm{~mm})$. The concentration of harmine and its hydrochloride salt were $1.35 \mu \mathrm{M}$ in methanol. Ammonium chloride was prepared at $200 \mathrm{mM}$ in methanol. Harmine/ammonium chloride solutions were prepared as follows: aliquots of ammonium chloride solution were added to the harmine solution ( $3.5 \mathrm{~mL}, 4.725 \mathrm{nmol}$ ) that were $0.5 \mu \mathrm{L} \mathrm{NH}_{4} \mathrm{Cl}$ solution (0.1 $\mu$ mol, $20 \mathrm{eq}$ mol to harmine), $10 \mu \mathrm{L} \mathrm{NH} \mathrm{NH}_{4} \mathrm{Cl}$ solution ( $2 \mu \mathrm{mol}, 400 \mathrm{eq}$ mol to harmine), $20 \mu \mathrm{L}$ $\mathrm{NH}_{4} \mathrm{Cl}$ solution ( $4 \mu \mathrm{mol}, 800$ eq mol to harmine), 30 $\mu \mathrm{L} \mathrm{NH}_{4} \mathrm{Cl}$ solution ( $6 \mu \mathrm{mol}, 1200$ eq mol to harmine), and $50 \mu \mathrm{L} \mathrm{NH}_{4} \mathrm{Cl}$ solution (10 $\mu \mathrm{mol}, 2000 \mathrm{eq} \mathrm{mol}$ to harmine).

Ultraviolet (UV) spectra were acquired with a V-550 UV-spectrometer (Jasco Co., Tokyo, Japan). The concentrations of harmine for the UV-spectra were 10 $\mu \mathrm{M}$ in methanol, and $10 \mu \mathrm{M}$ in the $\mathrm{HCl}$ acidic methanol/water solution (methanol:2 $\mathrm{N} \mathrm{HCl}$ aqueous solution $=50: 50[\mathrm{vol} / \mathrm{vol}]$ at $\mathrm{pH} 3.0)$. The estimations of the absorbance of all matrices at $337 \mathrm{~nm}$ were performed at $20 \mu \mathrm{M}$ final concentrations in methanol and $\mathrm{HCl}$ methanol solutions.

\section{Solid-Phase Fluorescence and Ultraviolet Spectra}

Solid-phase fluorescence and UV-spectra were acquired with a FP-6500 (Jasco Co., Tokyo, Japan) using a glass plate $(10 \times 40 \mathrm{~mm})$. Fluorescence spectra at the $300 \mathrm{~nm}$ excitation wavelength were obtained with 
Table 1. Structure of analytes

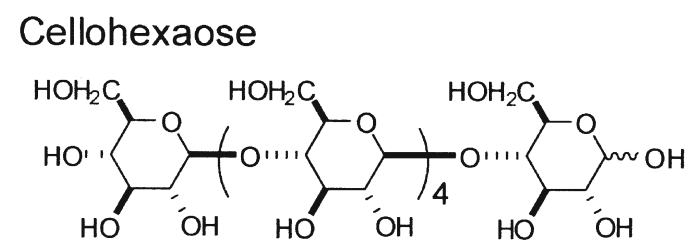

Cellohexitol<smiles>CC(O)[C@H](OC(C)(C)C1OC(CO)[C@@H](OC(C)(C)C2OC(CO)C(O)C(O)C2O)C(O)C1O)C(O)C(O)C(O)CO</smiles>

\section{Isomaltohexaose}<smiles>CC(C)OC[C@H]1C[C@@H](O)[C@@H](O)[C@H](O)O1</smiles>

\section{Gentiohexaose}

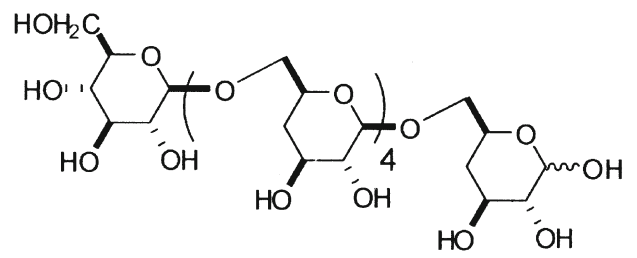

Lacto- $N$-tetraose (LNT)

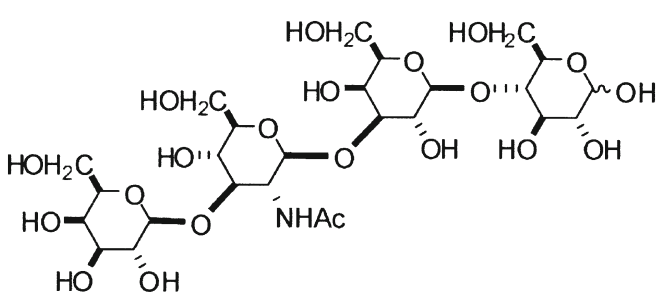

\section{$\mathrm{N}$-acetylchitohexaose}

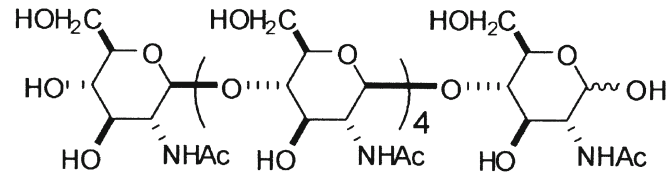

\section{Maltotriose}<smiles>CC1O[C@H](O)C(O)[C@H](O)[C@@H]1O[C@H]1OC(C)[C@H](O[C@H]2OC(CO)[C@@H](O)C(O)[C@H]2O)C(O)[C@H]1O</smiles>

Maltotetraose

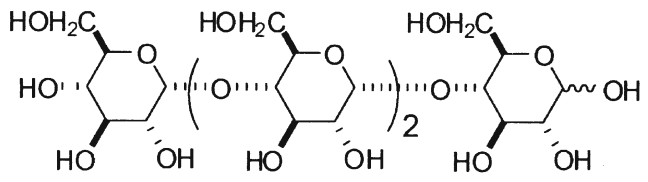

\section{Maltopentaose}

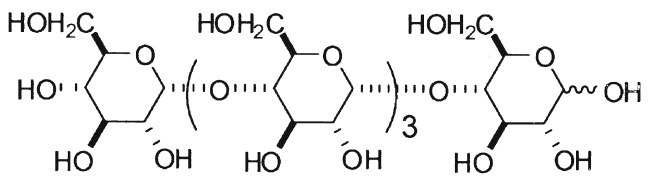

\section{Maltohexaose}

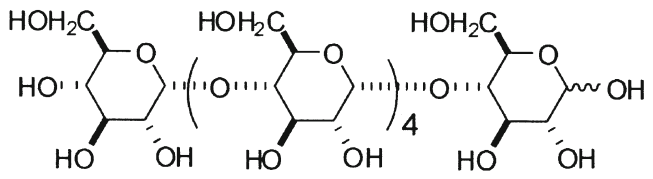

\section{Maltoheptaose}

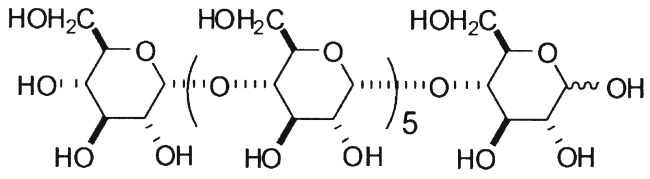

by normal detection with glass plates. Standard matrices of harmine and harmine hydrochloride on the grass plates were prepared by a thin-layer method [16] in which a $2.5 \mu \mathrm{L}$ aliquot of $25 \mathrm{mM}$ harmine and harmine hydrochloride in methanol was deposited and dried up, and another aliquot of the matrix solution was deposited and dried up.

The mixed crystals for quantitative analyses of the fluorescence intensity were prepared on the glass plates by almost same procedures of the sandwich method as for the MALDI MS measurements; a $2.5 \mu \mathrm{L}$ aliquot of 25 $\mathrm{mM}$ harmine methanol solution was deposited into the center of an O-ring on the grass plate, dried up, and an aliquot of $2.5 \mu \mathrm{L}$ of ammonium chloride was deposited and dried up. Finally, another aliquot of $2.5 \mu \mathrm{L}$ of the matrix solution was deposited and dried up. After removing the O-ring on the grass plate, we measured fluorescence spectra. 


\section{Results and Discussion}

\section{Sodium Chloride Versus Ammonium Chloride Versus Hydrochloride as a Chloride Donor}

We used three different additives coupled to a harmine matrix to make a comparison of the generation of chloride-anionized molecules of neutral oligosaccharides. The sodium chloride additive proved not to be a good chloride anion donor to the analytes, even as the amounts of sodium chloride were changed in the mixed crystal. These results can be explained on the basis of the ion stability of the complexes; in other words, the sodium cation $\left(\mathrm{Na}^{+}\right)$can coordinate the analytes more than chloride anion $\left(\mathrm{Cl}^{-}\right)$can [3]; this is a disadvantage to the negative-ion mode ionization. In MALDI, a chloride anion-rich plume may be essential for the production of labile, chloride-anionized molecules.

The chloride-anionized molecule yields were improved by the addition of ammonium chloride as shown in Figures 1 and 2. A hydrochloride $(\mathrm{HCl})$ solution also worked to improve the generation of the desired molecules. The ionization efficiency curves of cellohexaose between $\mathrm{NH}_{4} \mathrm{Cl}$ and $\mathrm{HCl}$ were almost the same (data not shown). Following these experiments, we turned to the properties of the mixed crystal surface consisting of a $\beta$-carboline matrix and ammonium chloride or hydrochloride, and we tried to relate their properties to the generation of the chloride-anionized molecules of neutral oligosaccharides.

\section{Amount of Ammonium Chloride}

Figure 1 shows the MALDI mass spectra of cellohexaose obtained for different amounts of ammonium chloride in the mixed crystals. Harmine was used as a matrix here. As the upper spectrum indicates, chloride-anionized molecules were rarely observed without the addition of ammonium chloride. The middle spectrum was taken with $5.4 \mathrm{nmol}$ $\mathrm{NH}_{4} \mathrm{Cl}$, corresponding to $0.2 \mathrm{eq}$ mol to matrix and 10 eq mol to analyte; they are the same measurement conditions reported [4]. The lower spectrum was obtained with $92.2 \mathrm{nmol} \mathrm{NH}{ }_{4} \mathrm{Cl}$, corresponding to $3.3 \mathrm{eq}$ mol to matrix. The relative abundance of the chlorideanionized molecule is highest in the latter spectra.

The ion abundance ratios [Analyte $+\mathrm{Cl}]^{-} /[$Matrix + $\mathrm{Cl}^{-}$were plotted as a function of the amounts of added ammonium chloride to estimate the ionization efficiency (Figure 2). All of the data in Figure 1 and 2 were taken under the same laser irradiation power. In this study, the sensitivity of ion detection is more improved than that of maltoheptaose reported previously [16], so that the chloride anionized molecules can be observed even under the lower irradiation laser power than that of the previous report [16]. The data points for all analytes in Figure 2 are fitted by logarithmic functions. The relative ion abundances rise for amounts of $\mathrm{NH}_{4} \mathrm{Cl}$ to matrix less than $\sim 4: 1$, at which point they almost

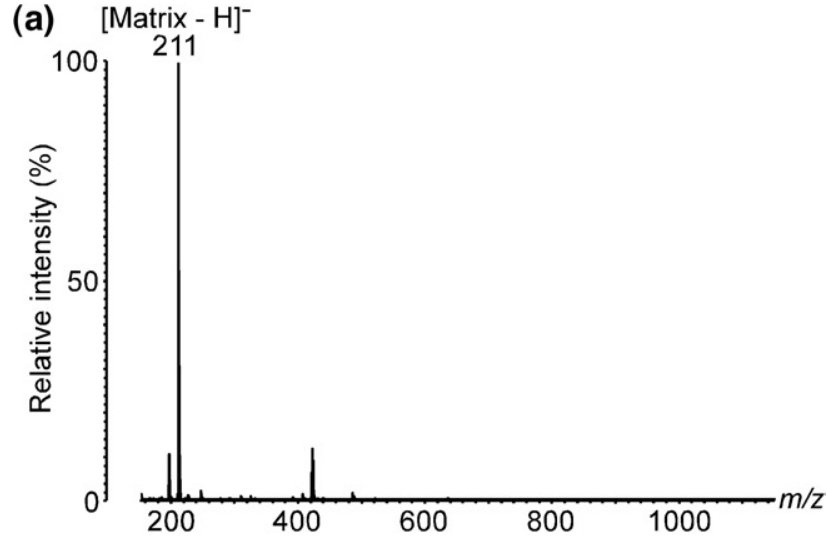

(b) $[\text { Matrix }-\mathrm{H}]^{-}$
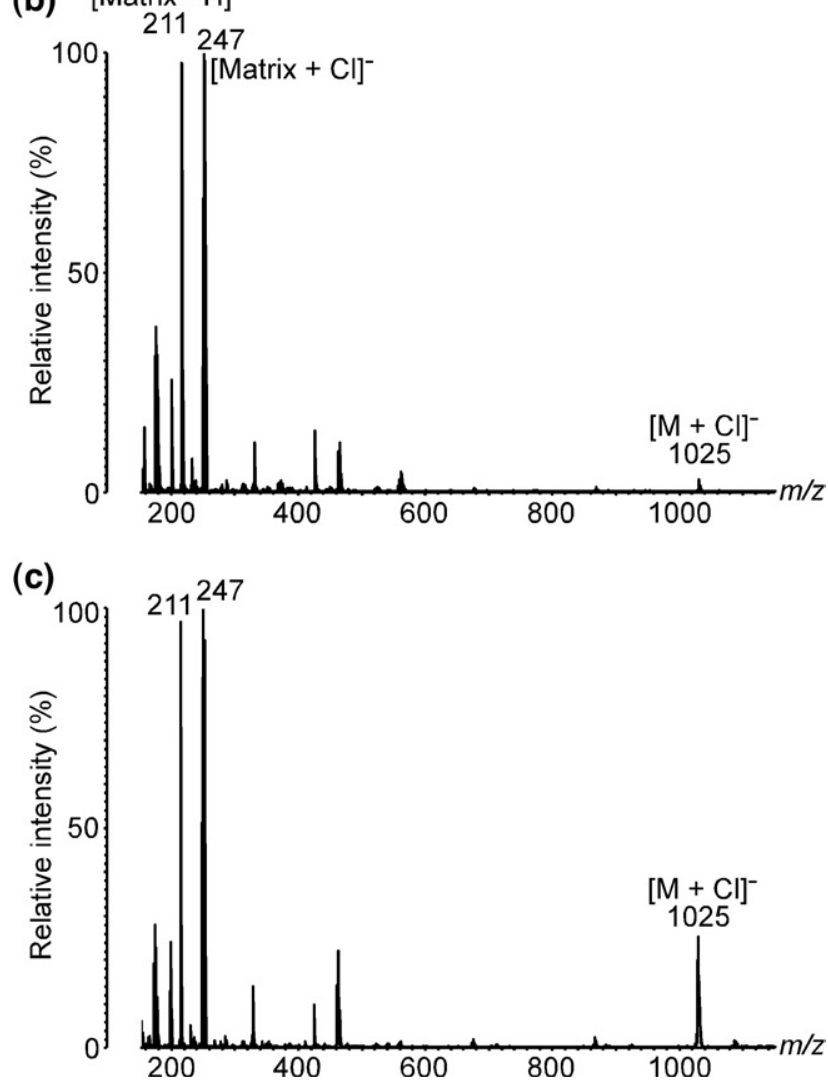

Figure 1. Negative-ion MALDI mass spectra of cellohexaose (a) using harmine as a matrix, (b) harmine with $5.4 \mathrm{nmol} \mathrm{NH}_{4} \mathrm{Cl}$ corresponding to 0.2 eq mol to matrix, and (c) harmine with 92.2 $\mathrm{nmol} \mathrm{NH}_{4} \mathrm{Cl}$ corresponding to $3.3 \mathrm{eq}$ mol to matrix.

saturate. This point is the optimum for the generation of chloride-anionized molecules in MALDI MS. In this matrix system, ammonium chloride works as co-matrix in MALDI like oligodeoxynucleotides' measurements [20-26].

The addition of the same amount of ammonium salts to matrix is known to improve the generation of the deprotonated molecules of oligodeoxynucleotides in the MALDI MS analyses. It was thought that the interaction between ammonium ions and multiply negative-charged analytes is the most important factor 


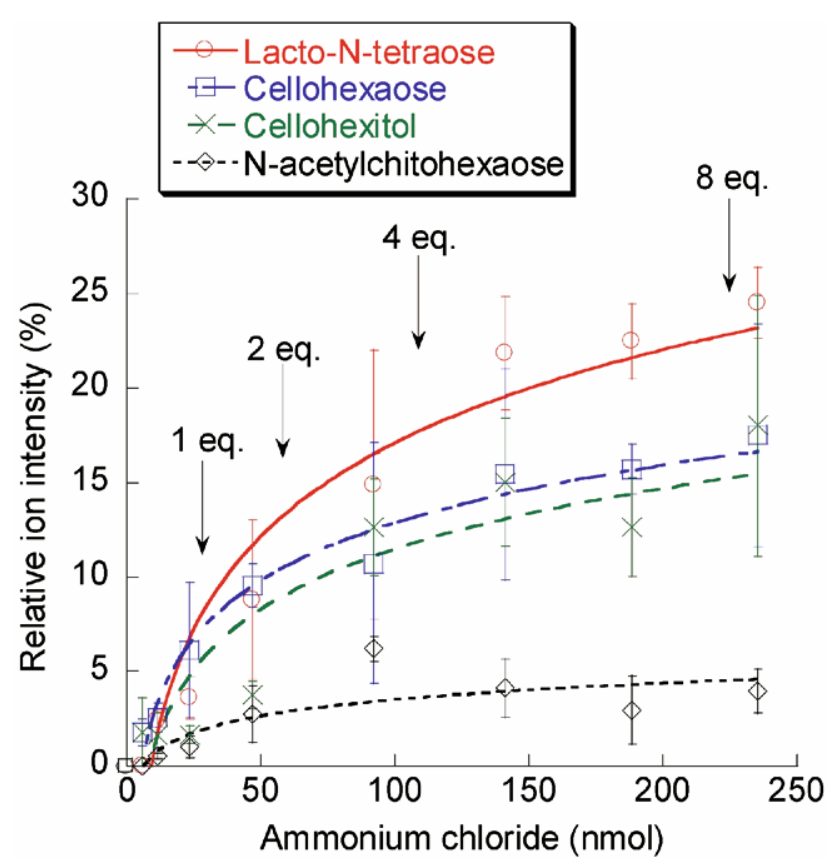

Figure 2. Ionization efficiency of lacto- $N$-tetraose (red circles), cellohexaose (blue squares), cellohexitol (green crosses), and $\mathrm{N}$-acetylchitohexaose (black diamonds). Ion abundance ratios of chloride-anionized molecules of analyte to matrix [Analyte + $\mathrm{Cl}^{-} /[\text {Matrix }+\mathrm{Cl}]^{-}$are plotted as a function of added ammonium chloride. These data points are fitted by logarithmic functions. The arrows annotate the molar ratios of amount of added ammonium chloride to matrix.

in the improvement of their MALD ionization. In the case of neutral oligosaccharides, the addition of ammonium chloride produced chloride-anionized molecules.

\section{Structures of Analytes \\ and Their Ionization Efficiency}

Several glucooligosaccharides linked by different glycosyl linkages in Table 1 , such as malto- $(\alpha 1 \rightarrow 4$ linkage), cello- $(\beta 1 \rightarrow 4)$, isomalto- $(\alpha 1 \rightarrow 6)$, and gentio- $(\beta 1 \rightarrow 6)$ hexaose, showed almost the same increasing tendency in the ion abundance ratios [analyte $+\mathrm{Cl}^{-} /[$matrix + $\mathrm{Cl}^{-}$as a function of the amounts of added ammonium chloride, a finding suggests that their ionization efficiency is not very dependent on their linkage types (data not shown). The results for maltooligosaccharides from triose to heptaose indicate that their relative ion abundance ratio to that of matrix is unrelated to oligosaccharide chain length (data not shown). Since the chloride anion may be attached to the hydroxyl group at the reducing terminal as a result of its acidity [4], we compared an analyte with its reduced analog such as cellohexaose and its hexitol; however, as shown in Figure 2, the increasing tendency in their ion abundance ratios to that of matrix do not differ significantly. In contrast, the abundance of the chloride-anionized molecule of lacto- $N$-tetraose (LNT) rises more rapidly than that of cellohexaose, and the ion abundance of lacto- $\mathrm{N}$ - tetraose is higher than that of cellohexaose (Figure 2). The $\mathrm{N}$-acetylglucosamine residue (GlcNAc) of LNT might have a higher affinity to chloride ion than does glucose because of its strong polarization at the acetoamide group. In contrast, $\mathrm{N}$-acetylchitohexaose showed the lowest ion abundance among the four sugar chains, although it consists of six GlcNAc. Because the molecule contained multi-affinity sites to chloride anion, the singly charged ion, which is commonly generated in MALDI MS, could be suppressed. The multi-charged ions were not detected in the spectrum of $\mathrm{N}$-acetylchitohexaose.

\section{Solid-Phase and Liquid-Phase Fluorescence Spectra}

Visible blue fluorescence was detected from the mixed crystals on the target plates upon $257 \mathrm{~nm}$ excitation. The intensity of the blue fluorescence increased with the amount of ammonium chloride added, a result suggesting that harmine formed a complex with ammonium chloride. Figure 3 shows the liquid-phase fluorescence spectra of harmine in methanol solution with changes in the amount of added ammonium chloride. The peak intensity from harmine at $365 \mathrm{~nm}$ decreased and a new peak appeared at $407 \mathrm{~nm}$ (Figure $3 \mathrm{~b}$ ). The peaks occurring at 365 and $407 \mathrm{~nm}$ came from the harmine hydrochloride in methanol solution (Figure 3a). The fluorescence at $407 \mathrm{~nm}$ originated from protonated harmine below pH 7.0 in aqueous solution [28]. Here, the molar ratios of added ammonium chloride to harmine were extremely high in their liquid-phase fluorescence spectra (Figure $3 b$ ), and did not correspond to increases in the ion abundance ratio of the chloride-anionized molecules (Figure 2). This behavior occurs because equilibrium, at least including harmine and harminium, exists in the methanol solution of the mixture of harmine and ammonium chloride.

Figure 4 shows the solid-phase fluorescence spectra of the surface of the mixed crystal, made of harmine and harmine hydrochloride. Harmine had a peak with its maximum intensity at $366 \mathrm{~nm}$; that of harmine hydrochloride was observed at $448 \mathrm{~nm}$, with no peak at 366 $\mathrm{nm}$. Hence, these substances were clearly distinguished by their unique peaks. Figure $4 \mathrm{~b}$ shows the fluorescence spectra of the mixed crystal in which the spectral changes depended on the amount of ammonium chloride. The quantitative analysis of the fluorescence indicates that the peak intensity at $448 \mathrm{~nm}$ from harmine hydrochloride rise logarithmically as a function of the amount of ammonium chloride (Figure 5), and is saturated at amounts of $\mathrm{NH}_{4} \mathrm{Cl}$ three to five times higher than that of harmine. This finding correlates quantitatively with the chloride-anionized molecule abundance ratios (Figure 2). Overall, these results suggest that ammonium chloride works as a co-matrix, and that the role of ammonium chloride is the formation of hydrochloride salt of matrix. Matrix hydrochloride salt is essential for the generation of the chloride-anionized molecules of neutral oligosaccharides, rather than the 

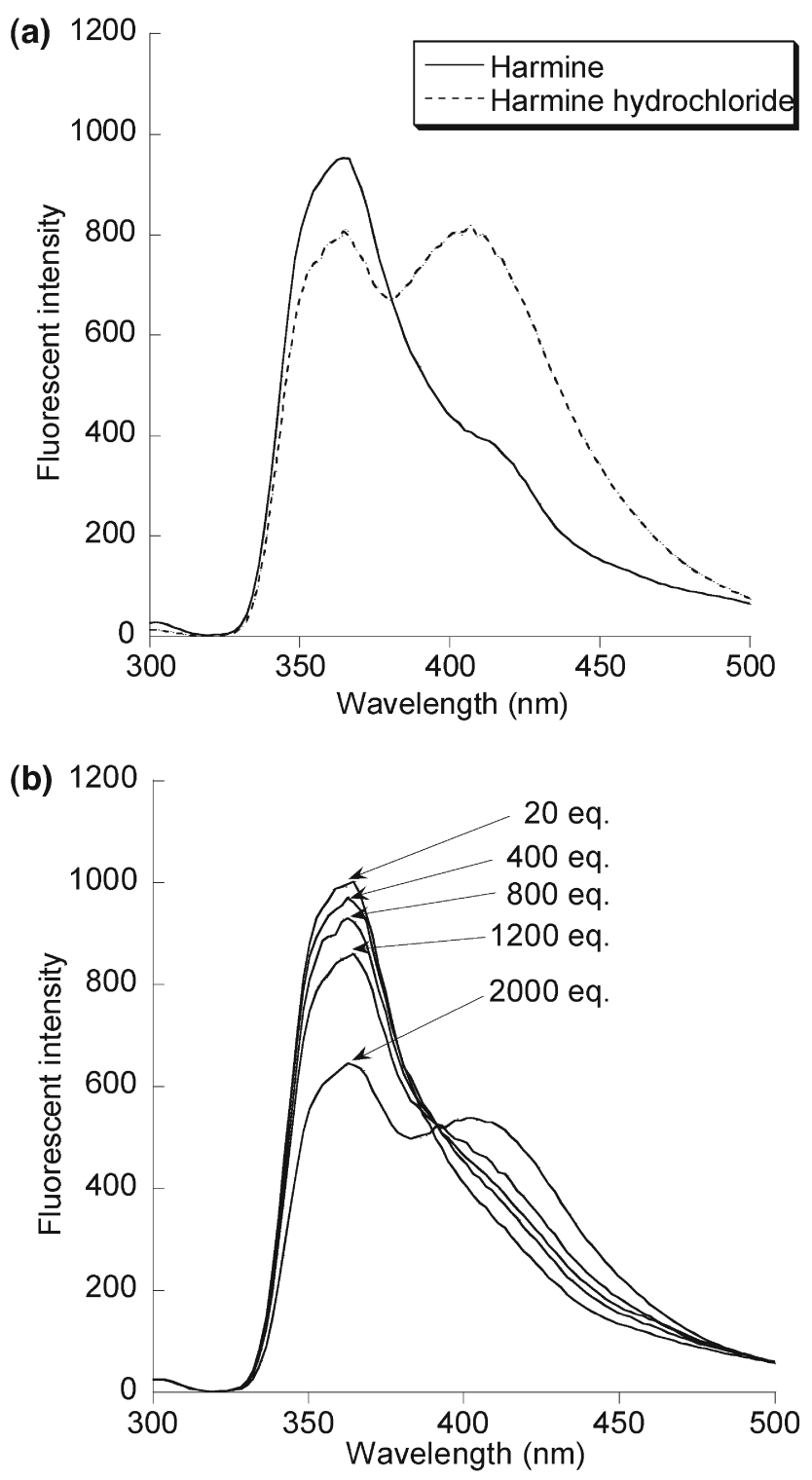

Figure 3. Liquid-phase fluorescence spectra of (a) harmine and harmine hydrochloride in methanol, and (b) harmine and $\mathrm{NH}_{4} \mathrm{Cl}$ mixed solutions in methanol.

coordination of ammonium ions to analytes that is a major reason for the improvement of the oligodeoxynucleotide ionization efficiency.

\section{Other $\beta$-Carboline Matrices}

The ion abundance ratios of the chloride-anionized molecules to matrices were compared with those for other $\beta$-carbolines under the same conditions (Table 2). $\beta$-Carboline hydrochlorides were used at the final concentration of $28.3 \mathrm{mM}$ in methanol without addition of ammonium chloride, and other $\beta$-carbolines were used at the same concentration, and $0.5 \mu \mathrm{L}$ aliquots of $100 \mathrm{mM}$ ammonium chloride aqueous solution were added in the preparation of mixed crystal. Harmine hydrochloride was the best matrix among the $\beta$-carboline hydrochlorides. The spectra obtained with harmine hydrochloride were much better in their signal-to-noise ratios than those obtained with harmine with three to five times higher amounts of ammonium chloride. However, in-source fragmentation took place easily in the case of harmine hydrochloride, even under low laser power.

\section{UV-Absorption Spectra of Harmine}

The solid-phase UV spectra of the matrix mixed crystal of harmine, harmine hydrochloride, and harmine with am-
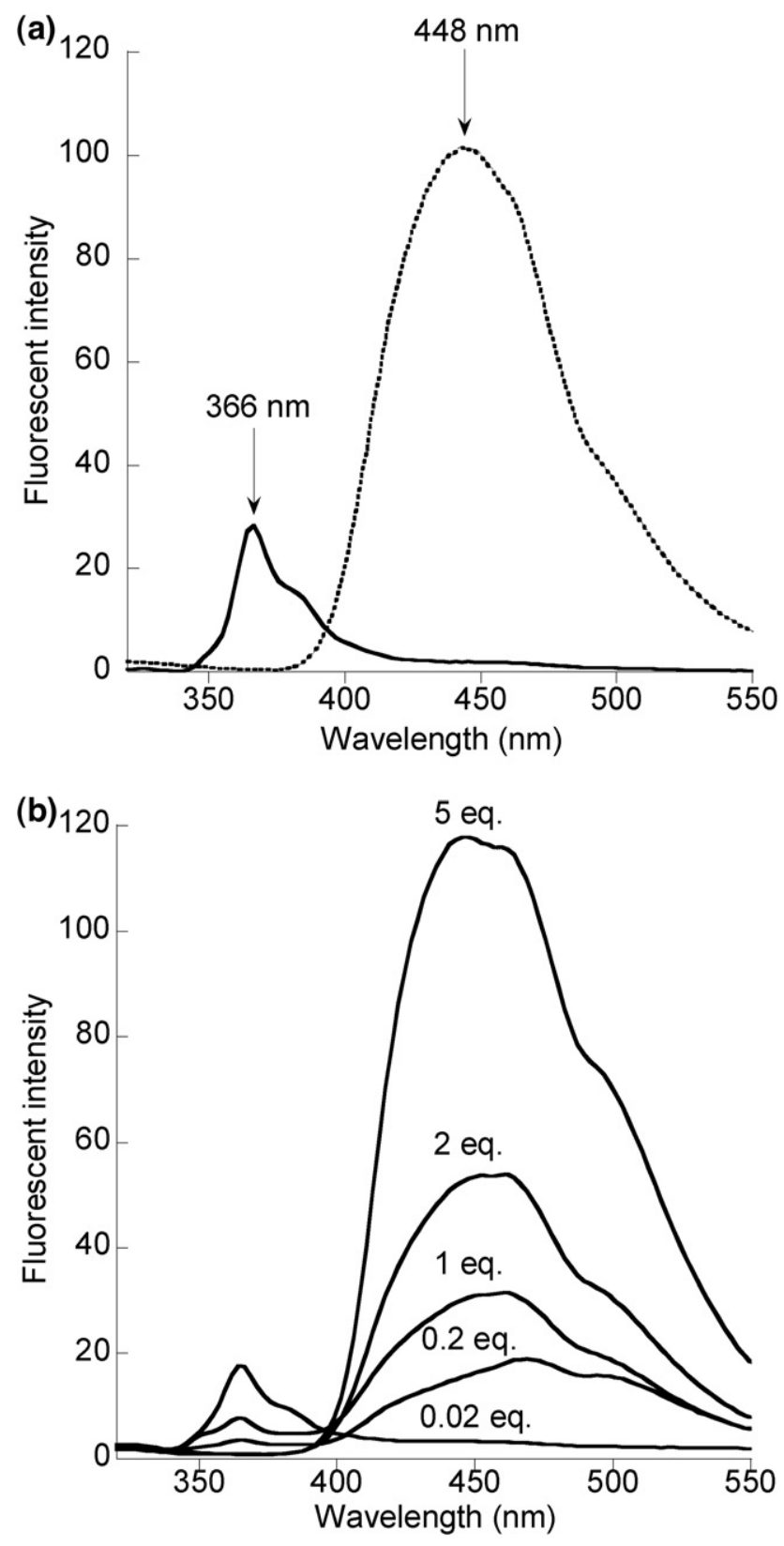

Figure 4. Solid-phase fluorescence spectra of (a) harmine (solid line) and harmine hydrochloride (dotted line) and (b) harmine$\mathrm{NH}_{4} \mathrm{Cl}$ mixed crystal on the grass plates. The molar ratios of ammonium chloride/harmine matrix to matrix (mol. eq.) were annotated. 


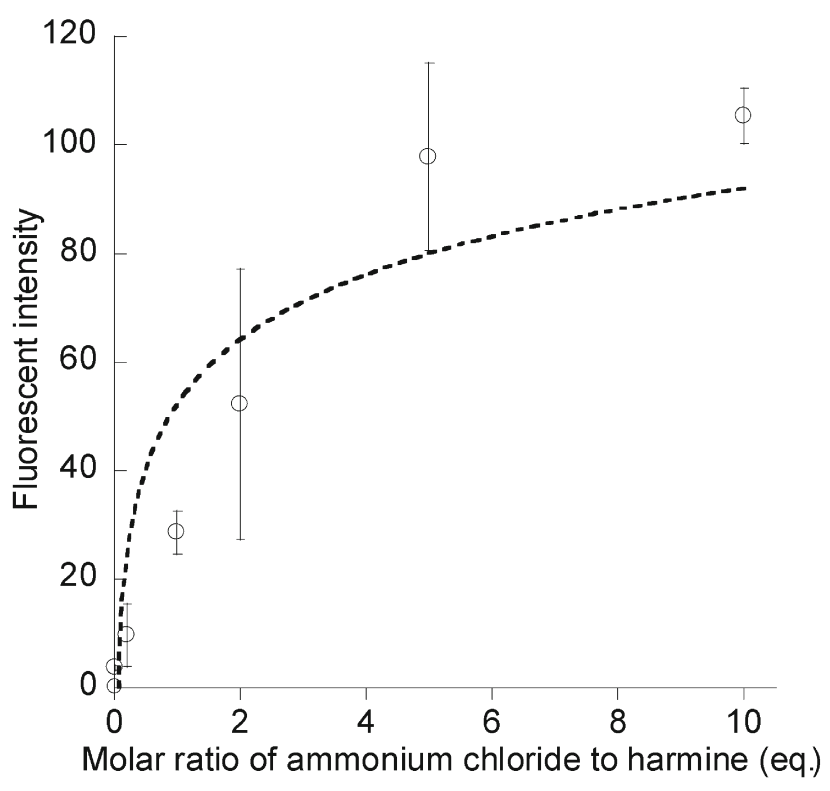

Figure 5. Fluorescence intensity at $448 \mathrm{~nm}$ of mixed crystals as a function of the amount of ammonium chloride. These data points are fitted by a logarithmic function. monium chloride were acquired by scanning of the reflection of the injection light. Their reflection spectra varied in every measurement because of their amorphous solid surfaces. The average light reflection of harmine hydrochloride was higher than that of harmine at the laser irradiation wavelength of $337 \mathrm{~nm}$ in MALDI. Ammonium chloride itself shows the $337 \mathrm{~nm}$ light reflection, and the reflection increases with the amount of ammonium chloride added in the harmine- $\mathrm{NH} 4 \mathrm{Cl}$ matrix system. Thus, we cannot relate these results to the ionization efficiency directly. In contrast, harminium in methanol absorbs 337 $\mathrm{nm}$ light more than harmine [29]. The liquid-phase UVabsorption spectra of harmine were measured in methanol and in $\mathrm{HCl}$-acidic methanol solution (Figure 6). Since the UV-spectra of harmine and harmine hydrochloride just dissolved in methanol are the same (data not shown), we measure the harmine in the $\mathrm{HCl}$ acidic methanol solution to obtain the spectrum of the protonated harmine form [29]. The absorption at $300 \mathrm{~nm}$ in harmine was shifted to close to $337 \mathrm{~nm}$ in the spectrum of harminium, a value which is the wavelength of the $\mathrm{N}_{2}$ laser used in the MALDI-MS. From the liquid-phase data, the matrix of

Table 2. Matrix structure and their ionization efficiency

\begin{tabular}{|c|c|c|}
\hline Matrix & Structure & $\begin{array}{c}\text { Ion abundance ratio (\%) of } \\
{[\text { Analyte }+\mathrm{Cl}]^{-} /[\text {Matrix }+\mathrm{Cl}]^{-\mathrm{a}}}\end{array}$ \\
\hline Harmine $\mathrm{HCl}$ & & 34.4 \\
\hline Harmine $+100 \mathrm{mM} \mathrm{NH}_{4} \mathrm{Cl}$ & & 10.8 \\
\hline Harmane $+100 \mathrm{mM} \mathrm{NH}_{4} \mathrm{Cl}$ & & 5.3 \\
\hline Norharmane $+100 \mathrm{mM} \mathrm{NH}_{4} \mathrm{Cl}$ & & 3.3 \\
\hline Harmaline $+100 \mathrm{mM} \mathrm{NH}_{4} \mathrm{Cl}$ & & 0 \\
\hline Norharmane $\mathrm{HCl}$ & & 0 \\
\hline Harmaline $\mathrm{HCl}$ & & 0 \\
\hline
\end{tabular}

alonization efficiency is estimated from ion abundance ratios of chloride-anionized molecules of analytes to that of harmine $\left[\mathrm{Analyte}+\mathrm{Cl}^{-} /\left[\mathrm{Matrix}+\mathrm{Cl}^{-}\right.\right.$. $\beta$-Carboline hydrochlorides are used without addition of ammonium chloride at the final concentration of $28.3 \mathrm{mM}$ in methanol, and other $\beta$-carbolines are used in the same concentration and $0.5 \mu \mathrm{L}$ aliquots of $100 \mathrm{mM}$ ammonium chloride aqueous solution are added in the preparation of mixed crystal. 


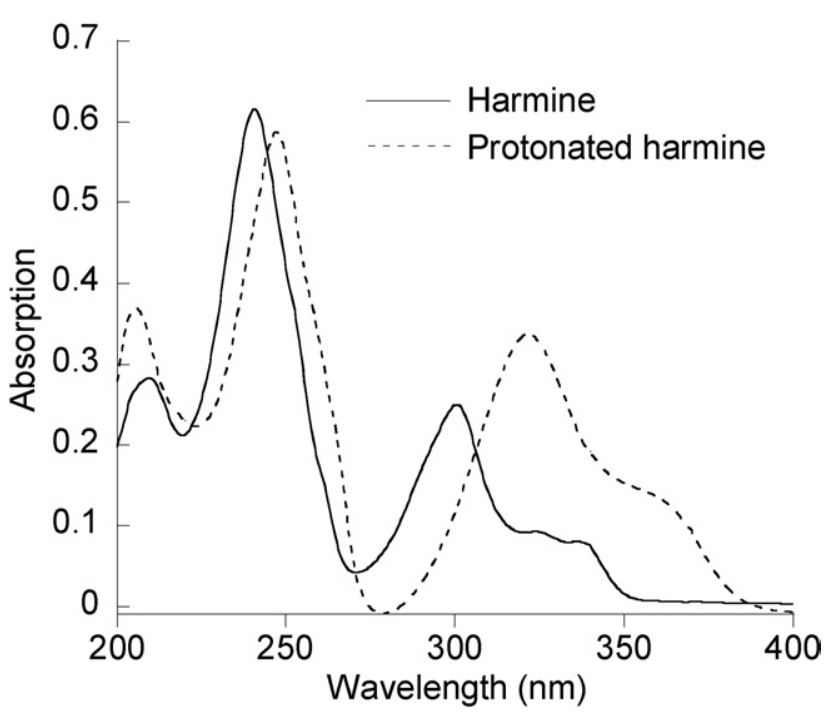

Figure 6. UV-absorbance spectra of harmine in methanol with or without $\mathrm{HCl}$.

harmine with ammonium chloride and its hydrochloride salt are excited by the MALDI laser more effectively than the harmine only. The liquid-phase UV data are not consistent with the solid-phase data.

\section{Principal Factors in the Ionization Efficiency}

What prime factor or factors govern the ionization efficiency in the generation of chloride-anionized molecules of neutral oligosaccharides in MALDI? First, the formation of matrix-hydrochloride complexes is required for a high chloride anion yield. A chloride anion coordinated to a protonated matrix easily desorbs and ionizes. Thus, the formation of harmine hydrochloride in the mixed crystal is directly related to the ionization efficiency and the ion abundance of the chloride-anionized molecules of neutrals. This point differs from the principal role of the co-matrix in improvement of the oligodeoxynucleotide ionization [26].

\section{Conclusions}

To generate chloride-anionized molecules $[\mathrm{M}+\mathrm{Cl}]^{-}$by MALD ionization, the optimal amount of ammonium chloride is around four times that of the matrix. Their respective solid-phase fluorescence spectra indicate that harmine with a large amount of ammonium chloride converts to the hydrochloride in the mixed crystal and that the amount of harmine hydrochloride correlates well to the ion abundance ratios of analytes to that of the matrix. The matrix hydrochloride form is essential for the production of chloride-anionized molecules. These results indicate that the roles of ammonium chloride as a co-matrix in the generation of chlorideanionized molecules of neutral oligosaccharides differs from that of deprotonated molecules of multiply negative-charged oligodeoxynucleotides.
Solid-phase spectroscopy allows us to analyze the mixed crystal matrix surface on the target, and we identify the formation of the matrix hydrochlorides from their solid-phase fluorescence. The ionization efficiency or relative abundances of the chloride-anionized molecules correlate quantitatively with the solid-phase fluorescence intensity at $448 \mathrm{~nm}$ from the matrix hydrochloride, but not with those from the liquid-phase fluorescence spectra. The solid-phase spectroscopic analysis of the matrix mixed crystals is essential to study the mechanism of the MALDI process, and can be useful in screening for new effective matrices and/or couples of matrix and additives, or for co-matrices for MALD ionization.

\section{Acknowledgments}

The authors thank Ms. Ayako Morishima (Jasco Co., Japan) for the measurements of solid-phase fluorescence spectra and for helpful discussions.

\section{References}

1. Stahl, B.; Steup, M.; Karas, M.; Hillenkamp, F. Analysis of Neutral Oligosaccharides by Matrix-Assisted Laser Desorption/Ionization Mass Spectrometry. Anal. Chem. 1991, 63, 1463-1466.

2. Strupat, K.; Karas, M.; Hillenkamp, F. 2,5-Dihydroxybenzoic Acid: A New Matrix for Laser Desorption-Ionization Mass Spectrometry. Int. J. Mass Spectrom. Ion Processes 1991, 111, 89-102.

3. Karas, M.; Glückmann, M.; Schäfer, J. Ionization in Matrix-Assisted Laser Desorption/Ionization: Singly Charged Molecular Ions are the Lucky Survivors. J. Mass Spectrom. 2000, 35, 1-12.

4. Cai, Y.; Jiang, Y.; Cole, R. B. Anionic Adducts of Oligosaccharides by Matrix-Assisted Laser Desorption/Ionization Mass Spectrometry. Anal. Chem. 2003, 75, 1638-1644.

5. Breuker, K.; Knochenmuss, R.; Zenobi, R. Matrix-Assisted Laser Desorption/Ionization: With Reagent Ion Generation Directly from a Liquid Matrix. Int. J. Mass Spectrom. 1998, 176, 149-159.

6. Nonami, H.; Fukui, S.; Erra-Balsells, R. $\beta$-Carboline Alkaloids as Matrices for Matrix-Assisted Ultraviolet Laser Desorption Time-of-Flight Mass Spectrometry of Proteins and Sulfated Oligosaccharides: A Comparative Study Using Phenylcarbonyl Compounds, Carbazoles, and Classical Matrices. J. Mass Spectrom. 1997, 32, 287-296.

7. Nonami, H.; Tanaka, K.; Fukuyama, Y.; Erra-Balsells, R. $\beta$-Carboline Alkaloids as Matrices for UV-Matrix-Assisted Laser Desorption/ Ionization Time-of-Flight Mass Spectrometry in Positive and Negative Ion Modes. Rapid Commun. Mass Spectrom. 1998, 12, 285-296.

8. Nonami, H.; Wu, F.; Thummel, R. P.; Fukuyama, Y.; Yamaoka, H.; Erra-Balsells, R. Evaluation of Pyridoindoles, Pyridylindoles, and Pyridylpyridoindoles as Matrices for Ultraviolet Matrix-Assisted Laser Desorption/Ionization Time-of-Flight Mass Spectrometry. Rapid Commun. Mass Spectrom. 2001, 15, 2354-2373.

9. Wong, A. W.; Cancilla, M. T.; Voss, L. R.; Lebrilla, C. B. Anion Dopant for Oligosaccharides in Matrix-Assisted Laser Desorption/Ionization Mass Spectrometry. Anal. Chem. 1999, 71, 205-211.

10. Wong, A. W.; Wong, H.; Lebrilla, C. B. Selection of Anionic Dopant for Quantifying Desialylation Reactions with MALDI-FTMS. Anal. Chem. 2000, 72, 1419-1425.

11. Chai, W. G.; Piskarev, V.; Lawson, A. M. Negative-Ion Electrospray Mass Spectrometry of Neutral Underivatized Oligosaccharides. Anal. Chem. 2001, 73, 651-657.

12. Pfenninger, A.; Karas, M.; Finke, B.; Stahl, B. Structural Analysis of Underivatized Neutral Human Milk Oligosaccharides in the Negative Ion Mode by Nano-Electrospray MS ${ }^{\text {; }}$ Part 1, Methodology. J. Am. Soc. Mass Spectrom. 2002, 13, 1331-1340.

13. Yamagaki, T.; Nakanishi, H. Negative-Mode Matrix-Assisted Laser Desorption/Ionization Mass Spectrometry of Maltoheptaose and Cyclomaltooligosaccharides. J. Mass Spectrom. Soc. Jpn. 2002, 50, 204-207.

14. Yamagaki, T.; Suzuki, H.; Tachibana, K. In-Source and Post-Source Decay in Negative-Ion Matrix-Assisted Laser Desorption/Ionization Time-of-Flight Mass Spectrometry of Neutral Oligosaccharides. Anal. Chem. 2005, 77, 1701-1707.

15. Yamagaki, T.; Suzuki, H.; Tachibana, K. A Comparative Study of the Fragmentation of Neutral Lactooligosaccharides in Negative-Ion Mode by UV-MALDI-TOF and UV-MALDI Ion-Trap/TOF Mass Spectrometry. J. Am. Soc. Mass Spectrom. 2006, 17, 67-74.

16. Suzuki, H.; Yamagaki, T.; Tachibana, K. Optimization of Matrix and Ammonium Chloride Additive for Effective Ionization of Neutral 
Oligosaccharides as Chloride Ion Adducts in Negative-Mode MALDI-TOF Mass Spectrometry. J. Mass Spectrom. Soc. Jpn. 2005, 53, 227-229.

17. Zhu, J.; Cole, R. B. Ranking of Gas-Phase Acidities and Chloride Affinities of Monosaccharides and Linkage Specificity in CollisionInduced Decompositions of Negative Ion Electrospray Generated Chloride Adducts of Oligosaccharides. J. Am. Soc. Mass Spectrom. 2001, 12 , 1193-1204.

18. Jiang, Y.; Cole, R. B. Oligosaccharide Analysis Using Anion Attachment in Negative Mode Electrospray Mass Spectrometry. J. Am. Soc. Mass Spectrom. 2005, 16, 60-70.

19. Cai, Y.; Cole, R. B. Stabilization of Anionic Adducts in Negative Ion Electrospray Mass Spectrometry. Anal. Chem. 2002, 74, 985-991.

20. Currie, G. J.; Yates, J. R. Analysis of Oligodeoxynucleotides by NegativeIon Matrix-Assisted Laser Desorption Mass Spectrometry. J. Am. Soc. Mass Spectrom. 1993, 4, 955-963.

21. Pieles, U.; Zurcher, W.; Schar, M.; Moser, H. E. Matrix-Assisted Laser Desorption Ionization Time-of-Flight Mass Spectrometry: A Powerful Tool for the Mass and Sequence Analysis of Natural and Modified Oligodeoxynucleotides. Nucleic Acids Res. 1993, 21, 3191-3196.

22. Vandell, V. E.; Limbach, P. A. Polyamine Co-matrices for MatrixAssisted Laser Desorption/Ionization Mass Spectrometry of Oligonucleotides. Rapid Commun. Mass Spectrom. 1999, 13, 2014-2021.

23. Simmons, T. A.; Limbach, P. A. Influence of Co-matrix Proton Affinity on Oligodeoxynucleotide Ion Stability in Matrix-Assisted
Laser Desorption/Ionization Time-of-Flight Mass Spectrometry. J. Am. Soc. Mass Spectrom. 1998, 9, 668-675.

24. Simmons, T. A.; Limbach, P. A. The Use of a Co-matrix for Improved Analysis of Oligodeoxynucleotides by Matrix-Assisted Laser Desorption/ Ionization Time-of-Flight Mass Spectrometry. Rapid Commun. Mass Spectrom. 1997, 11, 567-572.

25. Cheng, S.-W.; Chan, T.-W. D. Use of Ammonium Halides as Co-matrices for Matrix-Assisted Laser Desorption/Ionization Studies of Oligonucleotides. Rapid Commun. Mass Spectrom. 1996, 10, 907-910.

26. Asara, J. M.; Allison, J. Enhanced Detection of Phosphopeptides in Matrix-Assisted Laser Desorption/Ionization Mass Spectrometry Using Ammonium Salts. J. Am. Soc. Mass Spectrom. 1999, 10, 35-44.

27. Vorm, O.; Roepstorff, P.; Mann, M. Improved Resolution and Very High Sensitivity in MALDI TOF of Matrix Surfaces Made by Fast Evaporation. Anal. Chem. 1994, 66, 3281-3287.

28. Dias, A.; Varela, A. P.; Miguel, M. G.; Becker, R. S.; Burrows, H. D.; Macanita, A. L.; Becker, R. S. $\beta$-Carbolines. 2. Rate Constants of Proton Transfer from Multiexponential Decays in the Lowest Singlet Excited State of Harmine in Water as a Function of pH. J. Phys. Chem. 1996, 100, 17970-17977.

29. Dias, A.; Varela, A. P.; Miguel, M. G.; Macanita, A. L.; Becker, R. S. $\beta$-Carboline Photosensitizers. 1. Photophysics, Kinetics, and ExcitedState Equilibrium in Organic Solvents, and Theoretical Calculations. J. Phys. Chem. 1992, 96, 10290-10296. 